\title{
Representações sociais da droga e vulnerabilidade de usuários de CAPSad em relação às DST/ HIV/ AI DS
}

\section{Social representation of drugs and vulnerability of CAPSad users to HIV/ AI DS}

\author{
Andréia I sabel Giacomozzi* \\ Universidade Federal de Santa Catarina - UFSC, Florianópolis, Santa Catarina, \\ Brasil
}

\begin{abstract}
RESUMO
Esta pesquisa objetivou identificar as representações sociais do álcool e outras drogas, bem como as vulnerabilidades com relação às DST/HIV/AIDS de usuários de um CAPSad de um município do Sul do Brasil. Participaram 87 usuários. Utilizou-se o questionário estruturado e auto-administrado. Os resultados evidenciaram uma multiplicidade de fatores de risco às DST/HIV/AIDS entre os usuários, pois apesar de uma atitude favorável ao uso do preservativo, na prática houve baixa adesão ao uso do mesmo, baixo conhecimento sobre transmissão do HIV e importante presença de DSTs em suas histórias de vida. Além disso, observaram-se especificidades com relação às vulnerabilidade dos usuários do CAPSad com relação ao HIV/AIDS, pois o estar sobre efeito de alguma substância psicoativa foi fator dificultador da utilização do preservativo. Por outro lado, nas evocações sobre álcool/drogas surge a importante associação destas com sexo, diversão e prazer, o que indica que esta combinação pode corresponder a sexo desprotegido.
\end{abstract}

Palavras-chave: Representação social; AIDS; Drogas; Vulnerabilidade.

\begin{abstract}
The aim of the present research was to identify alcohol and other drugs social representations, as well as vulnerabilities related to STD/HIV/AIDS in a group of people attending a mental health public institution (CAPSad Psychosocial Attention Center for Alcohol \& Other Drugs) in Southern Brazil. 87 users participated in the study by answering a structured selfadministered questionnaire. Results showed several risk factors associated with STD/HIV/Aids among user: in spite of a positive attitude towards condoms, the study found a low rate of actual use of this preventive device, poor knowledge about HIV transmission and the significant role played by STDs in the users' lives. Moreover, CAPSad users present specific vulnerabilities related to HIV/Aids: being under the effect of a psychoactive substance made condom use less likely. On the other hand, alcohol and other drugs evocations are associated with sex, fun and pleasure, indicating that this combination may correspond to unsafe sex.

Keywords: Social representations; Aids; Drugs; Vulnerability.
\end{abstract}




\section{Introdução}

De acordo com a Organização Mundial de Saúde (OMS, 2001), cerca de $10 \%$ das populações dos centros urbanos de todo o mundo, consomem abusivamente substâncias psicoativas independentemente da idade, sexo, nível de instrução e poder aquisitivo. Sem considerar o uso de substâncias psicoativas de caráter ilícito, e considerando qualquer faixa etária, o uso indevido de álcool e tabaco tem a maior prevalência global, trazendo também as mais graves conseqüências para a saúde pública mundial. Estudo realizado por Murray e Lopez (1996) sobre a carga global de doenças estimou que o álcool seria responsável por cerca de $1,5 \%$ de todas as mortes no mundo, bem como sobre $2,5 \%$ do total de anos vividos em incapacidade, incluindo transtornos físicos (cirrose hepática, miocardiopatia alcoólica, etc.) e lesões decorrentes de acidentes (industriais e automobilísticos, por exemplo) influenciados pelo uso indevido de álcool.

Com relação à região sul do Brasil, dados do "II Levantamento Domiciliar sobre Uso de Drogas no Brasil", realizado pelo CEBRID, em 2005 (CARLINI, et al., 2007), que pesquisou em 108 cidades brasileiras de mais de 200 mil habitantes, mostram que nesta região o "uso na vida" das drogas (que não álcool e tabaco) foi de 14,8\%. Já o "uso na vida" do álcool foi de 73,9\% e a "dependência" do álcool é de $9,0 \%$ da população. O Sul apresenta índice significativo de "consumo" de maconha (9,7\%), de cocaína $(3,1 \%)$ e de crack $(1,1 \%)$. O índice de "dependência" da maconha é alto $(1,1 \%)$. Tomando os dados da referida pesquisa para a Região Sul, e considerando como referência a população de Florianópolis, chegarse-ia a um número estimado em torno de 35 mil dependentes de álcool e em torno de 4 mil dependentes de maconha neste município. O uso de drogas, inclusive álcool e tabaco, tem relação direta e indireta com uma série de agravos à saúde, entre os quais os acidentes de trânsito, as agressões, depressões clínicas e distúrbios de conduta, além de comportamento de risco no âmbito sexual e a transmissão do HIV pelo uso de drogas injetáveis e outros problemas de saúde decorrentes dos componentes da substância ingerida, e das vias de administração (MINISTÉRIO DA SAÚDE, 2003). No que tange a relação com comportamentos sexuais arriscados, vários estudos (TAQUETTE; ANDRADE; VILHENA; PAULA, 2005; COOK; POLLOCK; RAO; CLARK, 2002; SCIVOLETTO; TSUJI; ABDO; QUEIROZ; ANDRADE; GATTAZ, 1999; TAPERT; AARONS; SEDLARE; BROWN, 2001) observaram uma associação entre o uso de bebidas alcoólicas e ou outras drogas e ter DST - Doença sexualmente transmissível.

Além disso, observa-se que enquanto o consumo de drogas injetáveis diminui, o de drogas mais baratas e com efeito semelhante aumenta significativamente. É o caso do crack, cujo uso mais do que dobrou 
nesta região, de $0,4 \%$ para $1,1 \%$, entre 2001 e 2007 . Estudos realizados no Brasil e no exterior mostram que a prevalência do vírus HIV entre usuários de crack é bem maior do que na população geral $(0,6 \%)$. Em Campinas (SP), o índice de infecção nas pessoas que usam a droga é de 7\%. Em Nova Iorque, Miami e São Francisco, a prevalência de HIV entre adultos jovens que consomem crack é ainda maior: 15,7\% (AZEVEDO et al., 2007).

O acesso de usuários de álcool e outras drogas aos serviços especializados tem sido descrito como baixo. Pesquisa realizada pelo Ministério da Saúde descreve que apenas 23\% de usuários de álcool e outras drogas procuram tratamento específico para a dependência (MINISTÉRIO DA SAÚDE, 2001). Soma-se a isso dados do relatório mundial sobre drogas do UNODC $(2008)^{1}$ que mostram que, em muitos países, os recursos para a segurança pública superam aqueles dedicados à saúde pública. Faz-se necessário, portanto, um maior enfoque na questão de álcool e outras drogas como questão de saúde, assim como garantias de ampliação do acesso desta população aos serviços de saúde, sensibilizando todos os profissionais envolvidos com a assistência para a temática e para seu manejo.

Com o objetivo de viabilizar as deliberações da III Conferência Nacional de Saúde Mental, e de normalizar a atenção a usuários de álcool e drogas, o Ministério da Saúde publicou portarias voltadas para a estruturação de rede de atenção específica a estas pessoas. A Portaria GM/336 de 19 de fevereiro de 2002 define normas e diretrizes para a organização de serviços que prestam assistência em saúde mental, tipo "Centros de Atenção Psicossocial - CAPS"incluídos aqui os CAPS voltados para o atendimento aos usuários de álcool e drogas, os CAPSad (MI NISTÉRIO DA SAÚDE, 2003).

Os CAPSad devem oferecer atendimento diário, sendo capazes de oferecer atendimento nas modalidades intensiva, semi-intensiva e não-intensiva, permitindo o planejamento terapêutico dentro de uma perspectiva individualizada de evolução contínua. A equipe de profissionais deve ser multidisciplinar e a rede proposta se baseia nestes serviços comunitários, apoiados por leitos psiquiátricos em hospital geral e outras práticas de atenção comunitária (ex.: internação domiciliar, participação comunitária na discussão dos serviços), de acordo com as necessidades da população-alvo.

Os cuidados em saúde (incluindo saúde mental) requerem uma abordagem ampliada, sendo significados na mediação de condições sociais, nas relações intrafamiliares, além do apoio social e comunitário. Além disso, o acolhimento como recurso terapêutico, o vínculo e a continuidade da atenção como possibilidade de ruptura com a exclusividade do núcleo biomédico na determinação do processo saúde-doença são forças das equipes multidisciplinares dos CAPS para a melhoria da qualidade de vida dos usuários. A partir daí, torna-se importante pensar na integralidade da atenção a estes 
usuários, considerando não apenas as questões relacionadas ao uso das substâncias psicoativas, mas também todo o entorno da problemática que envolve outras questões importantes, como as vulnerabilidades às DST/HIV/Aids.

O conceito de vulnerabilidade originou-se a partir das discussões sobre fator, grupo e comportamento de risco em relação à aids, mais especificamente das lacunas identificadas nestes discursos (AYRES; FRANÇA JR; CALAZANS; SALETTI FILHO, 1999) e teve por objetivo superar a idéia individualista a respeito das determinantes para a prevenção, onde o foco deixa de ser o indivíduo e passa a ser suas relações (CAMARGO; BERTOLDO, 2006). Adota-se neste estudo a noção de que a vulnerabilidade tem ligações com aspectos cognitivos (conhecimento e informações da epidemia, atitudes, percepção do risco), os sentimentos associados, e as condutas relacionadas com a doença (atitude frente 0 preservativo, experiência sexuais, comportamentos arriscados).

O conceito de atitudes é importante para se compreender as vulnerabilidades frente às DST/HIV/Aids, pois segundo Vala (2000) esse conceito tem ligação com os comportamentos, porque pretende ser mediador entre a forma de pensar e a forma de agir dos indivíduos, permitindo identificar o posicionamento de um indivíduo frente à realidade social. As atitudes envolvem o que as pessoas pensam, sentem e como gostariam de se comportar em relação a um determinado objeto. Esta relação entre os componentes de uma atitude pode ser de coerência ou não, pois é possível encontrar inconsistência entre as atitudes e os comportamentos expressos pelas pessoas (RODRIGUES, et al., 2002; LIMA, 1996).

A favorabilidade nas atitudes de estudantes frente 0 uso do preservativo foi observada em vários estudos (CAMARGO; BOTELHO, 2007; GIACOMOZZI, 2008; CAMARGO; BARBARÁ, 2004; MARTINS, et al., 2006). Porém no estudo de Camargo e Botelho, 2007, o consumo abusivo de álcool relacionou-se com atitude menos favorável à utilização do preservativo, indicando que a atitude desfavorável à saúde envolve posicionamentos semelhantes em diversas situações ou objetos que podem comprometer o bem-estar. Além disso, conhecer as representações sociais do álcool e de outras drogas de usuários do CAPS ad pode propiciar um melhor entendimento do lugar que estas substâncias ocupam na existência destes sujeitos, além de possibilitar um possível aprofundamento da relação entre o uso de drogas e a prática do sexo inseguro. Assim, a teoria das Representações sociais proposta por Moscovici (1981), foi escolhida para este estudo pela sua importância na análise de aspectos psicossocioculturais que permeiam o processo saúde/doença e as práticas sociais relativas ao mesmo, bem como pelos atos de comunicação social e fenômenos coletivos que contribuem para a formação de condutas e de normas que regem o pensamento social. 
As atitudes impregnam as representações sociais e estas últimas determinam as condutas desejáveis ou permitidas. A compreensão das representações sociais proporciona o acesso às justificativas utilizadas para orientar julgamentos e ações acerca do mundo e permite conhecer as estratégias dos seres humanos quando estão diante da tarefa de compreender fatos desconhecidos. Assim, reconstroem as situações como conhecimentos que regem o processo de comunicação e ação em relação aos fenômenos (MOSCOVICl, 1976).

De acordo com Barbará, Sachetti e Crepaldi, 2005, o conceito de atitudes e a teoria das representações sociais têm sido muito utilizados para explicar a complexa problemática da aids. As atitudes são especialmente estudadas em pesquisas de sondagem de opinião enquanto estudos das representações sociais da epidemia têm tido um papel importante para compreensão do modo como se age diante dela e da sua prevenção.

No campo das representações sociais, estudos relacionados às questões da prática de saúde são importantes, pois assumem toda sua significação, possibilitando a apreensão de processos e mecanismos pelos quais o sentido do objeto de estudo é construído pelos sujeitos concretos (FONSECA; AZEVEDO; ARAUTO, et al., 2007). Portanto, considera-se a RS como o sentido atribuído a um dado objeto pelo sujeito do senso comum, a partir das informações adquiridas na prática e nas suas relações cotidianas (MARKOVÁ, 2006). Sua construção acontece, assim, a partir da elaboração de informações de diferentes ordens, que são continuamente transformadas, recriadas, articulando instâncias, níveis e dimensões (MADEIRA, 2001).

Este estudo objetivou, portanto, investigar o conhecimento frente ao HIV/Aids, atitudes frente o uso do preservativo, bem como representações sociais da droga de usuários do serviço do CAPSad do município de Florianópolis, para melhor compreender as vulnerabilidades específicas desta população às DST, HIV/Aids.

\section{Método e caracterização da Pesquisa}

Tratou-se de estudo de natureza descritiva. O estudo foi exploratório, não randômico. Os participantes foram usuários do CAPS ad do município de Florianópolis, convidados a participar. O estudo foi aprovado pelo comitê de ética em pesquisa com seres humanos da UFSC sob o protocolo no 81/09. 


\section{Metodologia}

\subsection{Amostra}

Participaram 87 usuários do CAPS ad de Florianópolis, a média de idade foi de 35 anos com desvio padrão de 12 anos. Com relação ao sexo: $89,7 \%$ dos participantes era do sexo masculino e $10,3 \%$ feminino. No que tange a cidade de nascimento dos participantes, 40,5\% declara ter nascido em Florianópolis, 34,2\% em cidades do interior de Santa Catarina, e $25,3 \%$ em outros estados. E no que concerne ao mercado de trabalho, categoria mais citada foi a de desempregados $(57,4 \%)$. Em segundo lugar aparece a categoria de autônomo ou biscateiro, com 18,39\%. O restante dos participantes distribuiu-se entre categorias profissionais como vendas, funcionários públicos, pensionistas, aposentados, etc. Quanto ao estado civil, observou-se que a maior proporção (36\%) declarou ser solteiro, $29,1 \%$ separado, $25,6 \%$ casado, $5,8 \%$ namorado e 3,5\% viúvo.

\subsection{I nstrumento}

Foi utilizado um questionário estruturado e auto-administrado. As questões podem ser classificadas em dois grupos. O primeiro se refere às características individuais dos participantes: idade, escolaridade, profissão, comorbidades, experiência amorosa e sexual, experiência com uso/abuso de drogas, uso de preservativo, incidência de DST. O segundo grupo de questões diz respeito ao conhecimento ligado ao HIV/Aids e atitudes frente o preservativo e representações sociais do álcool/drogas.

\subsection{Procedimentos}

Os participantes foram convidados a participar diretamente pela pesquisadora. Para aqueles que deram seu consentimento solicitou-se que respondessem o questionário individualmente. Para os que manifestaram dificuldades em leitura/escrita foi disponibilizada a leitura do mesmo.

\subsection{Análise de dados}

As respostas fechadas foram colocadas em um banco de dados do software SPSS (pacote estatístico para as ciências sociais). Foram realizadas análises estatísticas descritivas e análises de associação entre as variáveis.

A questão aberta do questionário foi analisada com auxílio do software SPAD-T Systhème Portable pour l'Analyse des Données Textuelles - SPAD-T (LEBART; SALEM, 1988). Com este software é 
possível explorar o campo das atribuições através da análise de correspondência, pois ele executa uma análise textual que permite aplicar uma ampla variedade de procedimentos estatísticos, tais como diferentes versões da análise de correspondência e análise de agrupamento.

Para o estudo das representações sociais, os fatores da AFC podem ser interpretados como princípios organizadores que explicitam as similaridades e diferenças da representação social em diferentes grupos. Pode ser uma supra-estrutura, ideal para verificar variações no campo representacional de indivíduos com características diferentes (DOISE; CLEMENCE; LORENZI-CIOLDI, 1992; OLIVEIRA; AMÂNCIO, 2005).

\section{Resultados}

Com relação à escolaridade dos participantes, observou-se que a maior proporção $(48,3 \%)$ declarou ter o ensino fundamental incompleto, $14,9 \%$ ensino médio completo, igual percentual $(12,6 \%)$ declarou ter ensino fundamental completo e ensino médio completo, $6,9 \%$ declarou ter ensino superior incompleto, 3,4\% declarou não ter estudado (Ver Tabela 1).

Tabela 1. Escolaridade dos Participantes

\begin{tabular}{lr}
\hline & Escolaridade \\
\hline Ensino Fundarn ental Incom pleto & $48,3 \%$ \\
Ensino Médio Completo & $14,9 \%$ \\
Ensino Fundarn ental Completo & $12,6 \%$ \\
Ensino Médio Incompleto & $8,4 \%$ \\
Ensino Superior Incompleto & $6,9 \%$ \\
Nắo estudou & $3,4 \%$ \\
Ensino Superior Completo & $1,1 \%$ \\
Total & $100 \%$ \\
\hline
\end{tabular}

Quanto às substâncias utilizadas, observou-se que mais da metade dos participantes declararam utilizar várias substâncias $(56,3 \%)$, em segundo lugar ficou a declaração do uso do álcool $(25,3 \%)$, em terceiro a maconha, com $13,8 \%$ e em quarto lugar ficou o crack, com $4,6 \%$.

Tabela 2. Substâncias Utilizadas

\begin{tabular}{lr}
\hline \multicolumn{3}{c}{ Qual a substâncias que você utiliza (va)? } \\
\hline Várias & $56,3 \%$ \\
Áloool & $25,3 \%$ \\
Maconha & $13,8 \%$ \\
Crack & $4,6 \%$ \\
\hline
\end{tabular}

\subsection{Relações Amorosas e Sexuais}


Questionou-se aos usuários se eles têm parceiros fixos. A maior proporção $(57,5 \%)$ declarou não ter parceiro fixo no momento da pesquisa, enquanto $42,5 \%$ disse ter um parceiro fixo.

Tabela 3. Relacionamento Sexual nos últimos 12 meses

\begin{tabular}{lr}
\hline \multicolumn{2}{c}{ Você teve uma ou mais relaçöes sexuais nos últimos 12 meses ? } \\
\hline Nẫo & $11,6 \%$ \\
Sim , com a mesma pessoa & $47,7 \%$ \\
Sim , com mais de um a pessoa & $40,7 \%$ \\
Total & $100 \%$ \\
\hline
\end{tabular}

Com relação ao número de parceiros sexuais, de acordo com a Tabela 3 , observou-se que a maior proporção $(47,7 \%)$ afirmou ter tido relações sexuais com a mesma pessoa nos últimos 12 meses, enquanto $40,7 \%$ afirmou ter tido relações com mais de uma pessoa.

Tabela 4. Uso de preservativo nos últimos 12 meses

\begin{tabular}{lr}
\hline \multicolumn{2}{c}{ Il os últimos 12 meses você utilizou preservativo ? } \\
\hline & $34,90 \%$ \\
Nẫo & $36,10 \%$ \\
Sim, em todas as relaçỗes & $20,50 \%$ \\
Nẫo sei & $8,40 \%$ \\
Total & $100 \%$ \\
\hline
\end{tabular}

No que concerne ao uso do preservativo, 36,1\% declarou que utilizou preservativo em todas as relações sexuais que teve nos últimos 12 meses, $34,9 \%$ declarou não ter utilizado, enquanto $20,5 \%$ declararam ter utilizado em algumas relações e $8,4 \%$ declarou não saber se utilizou.

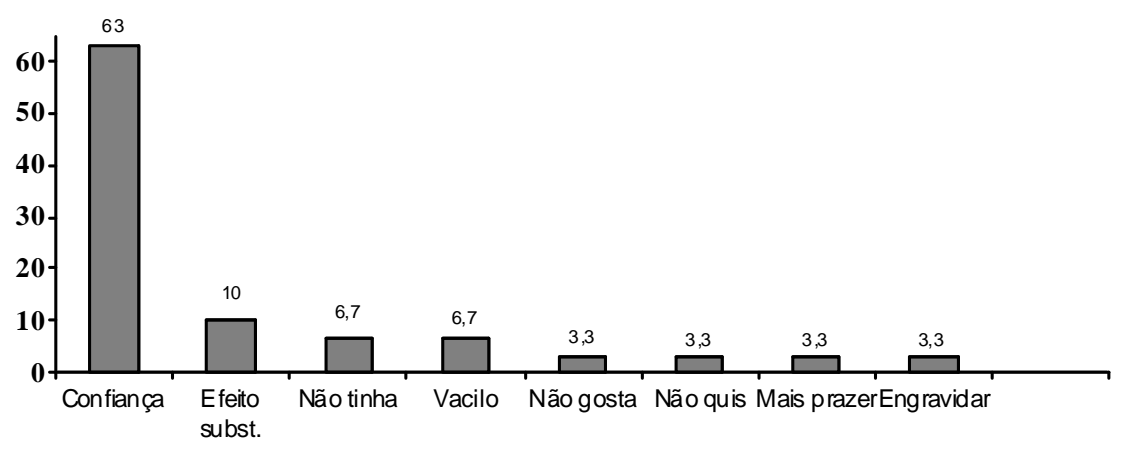

Figura 1- Motivos da não utilização do preservativo por usuários do CAPS nos últimos 12 meses.

Para os que declararam não ter utilizado preservativo em algum momento nos últimos 12 meses, questionou-se o motivo da não utilização. De acordo com a Figura 1, observa-se que em primeiro 
lugar, com $63 \%$ das respostas, surge a confiança no parceiro; $10 \%$ afirma que não utilizou porque estava sob efeito de álcool ou outras drogas, $6,7 \%$ por não ter consigo o insumo no momento e igual porcentagem por vacilo; 3,3\% não usou porque não gosta, não quis, para sentir mais prazer e para engravidar.

\subsection{Conhecimento e Prevalência de DST}

Questionou-se aos participantes, se eles sabem o que é uma DST e se já tiveram alguma destas doenças. A maioria $(73,6 \%)$ afirma saber o que é uma DST, enquanto $54 \%$ afirma nunca ter tido alguma doença sexualmente transmissível, $24,1 \%$ afirma já ter tido e $21,8 \%$ diz não saber se teve.

Para os que afirmaram já ter tido DST, questionou-se qual foi. De acordo com a Figura 2, observa-se que em primeiro lugar, com $75 \%$ de citações estão as Hepatites, em segundo lugar, a gonorréia com $10 \%$ e o HPV (10\%) e em terceiro lugar o chato, com $5 \%$ das respostas.

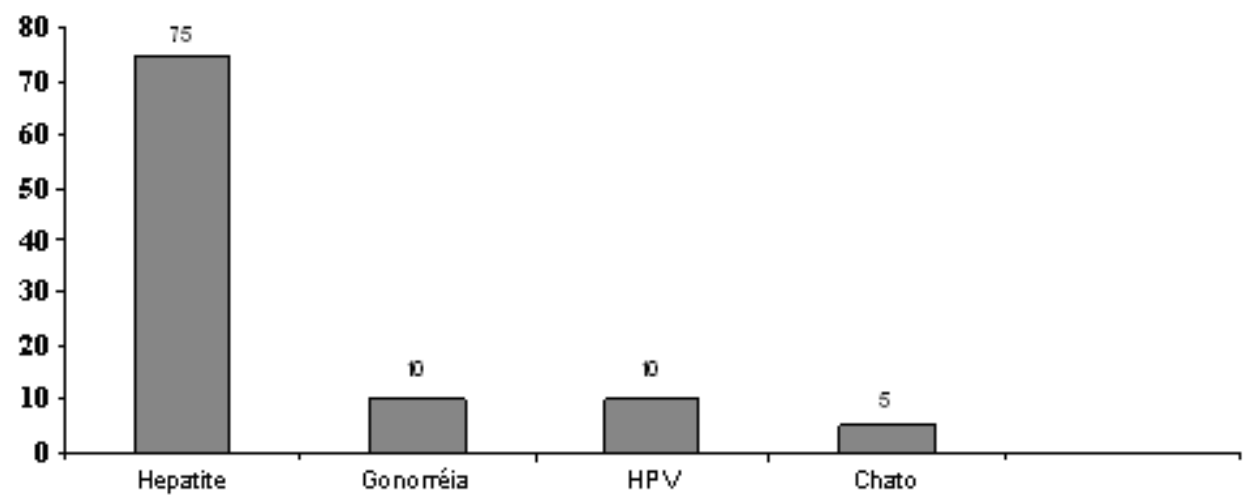

Figura 2. Tipos de DST

\subsection{Conhecimento sobre HIV/ Aids}

Perguntou-se se os participantes se eles se consideram bem informados sobre aids. A maior proporção $47,1 \%$ afirmou que se considera bem informado, enquanto $26,4 \%$ não se considera bem informado sobre aids e $26,4 \%$ não sabe responder.

Tabela 5. I nformação sobre aids

\begin{tabular}{lr}
\hline \multicolumn{3}{c}{ Você se considera bem informado sobre aids ? } \\
\hline Sim & $47,1 \%$ \\
Nẫo & $26,4 \%$ \\
Não sei & $26,4 \%$ \\
Total & $100 \%$ \\
\hline
\end{tabular}


Para verificar o conhecimento do grupo sobre aids, foi aplicado um sub-teste de conhecimento sobre a transmissão do vírus HIV (dimensão vírus e transmissão da aids) de Camargo, Barbará e Bertoldo (2005). A média de conhecimento do grupo foi de 5,12 (desvio padrão $=2,93$ ) dentre 10 possíveis.

Tal média de acerto se encontra abaixo da nota de corte de 7 acertos do sub-teste vírus da aids e transmissão da doença, parte integrante da medida sobre conhecimento acerca de aids, o que indica um baixo índice de conhecimento frente a doença.

\subsection{Atitude frente ao uso do preservativo}

Com relação à atitude frente o uso de preservativo, foi observado que os participantes obtiveram média de 3,61, numa escala de 1 atitude desfavorável - a 5 - atitude favorável - (desvio padrão 1,06). Por ser acima do ponto médio da escala, 3, percebe-se que o grupo posicionou-se favoravelmente ao uso do preservativo.

\subsection{Representações sociais do álcool e outras drogas}

Pediu-se aos participantes que escrevessem as 5 primeiras palavras que Ihes viessem a cabeça sobre álcool e outras drogas. As respostas primeiramente foram agrupadas em categorias e posteriormente procedeu-se com Análise Fatorial de Correspondência com auxílio do software SPAD.

A categoria mais citada pelos participantes com relação às drogas foi a que se referia à alegria/festas/diversão/prazer, com 48 ocorrências. $\mathrm{Em}$ segundo lugar, obteve-se a categoria angústia/depressão/tristeza/solidão, com 39 ocorrências, seguida pela lembrança das perdas/danos trazidos pelas drogas, com 34 citações. O mal-estar e outros sintomas físicos foram citados 17 vezes pelos participantes, e também a violência/morte/suicídio/ocorrências policiais (17), seguido pela fissura/vontade de usar ainda sentidos (13 citações). O relaxamento trazido pelas drogas (12) foi lembrado, mas também a vontade de parar e de não voltar mais ao uso (11), e logo em seguida tem-se a associação com o sexo e mulheres (9) e com insanidade/loucura (9) entre outros. 


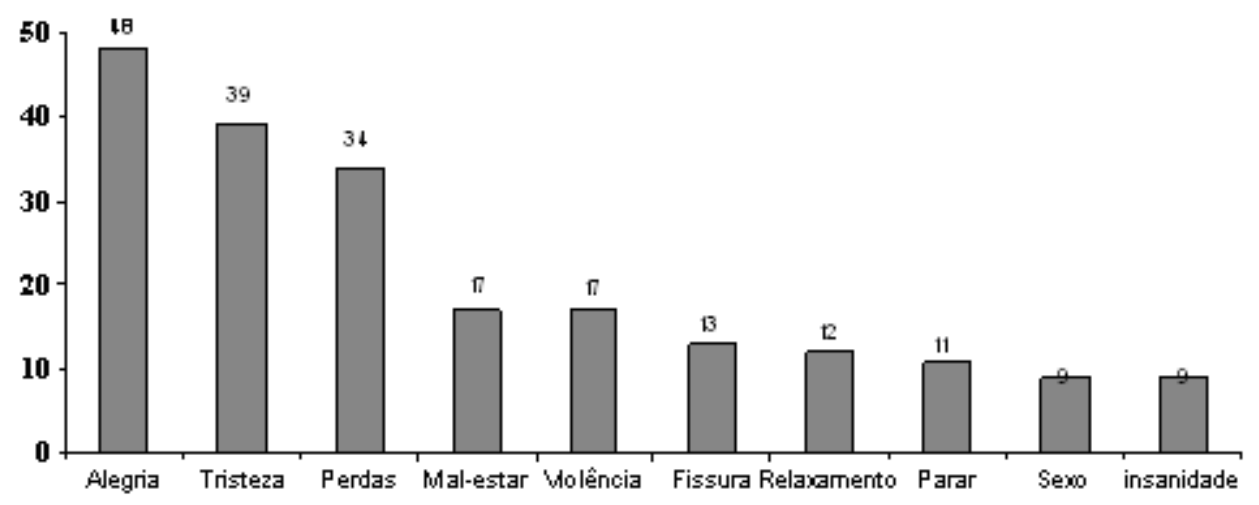

Figura 3. Representações Sociais do álcool e outras drogas

A partir da Análise Fatorial de Correspondência, obtiveram-se 2 fatores. O fator 1 explica $58,84 \%$ enquanto o fator 2 explica $41,16 \%$, perfazendo um total de $100 \%$ da variância total das respostas (A Tabela 6 mostra as palavras que mais contribuíram para os 2 fatores).

Com o primeiro fator surge a noção de que as drogas representam uma oportunidade de aliviar a ansiedade, mas trazem tristeza e arrependimento. Um primeiro pólo ligado a este fator, ao qual se associaram as variáveis Homem e Trabalha relaciona as drogas ao desabafo, ao barato trazido, mas também surge a noção do desespero, da recaída e da tristeza, associando as drogas a algo ruim. Este pólo também faz uma importante associação das drogas com sexo. Um segundo pólo ligado a este mesmo fator, ao qual se associaram as variáveis Mulher e Não trabalha, traz a noção do arrependimento frente as lembranças, e da preocupação com as conseqüências causadas na família. Surge também a relação das drogas com a comida, visto que em algumas situações acontece o aumento e em outras a perda do apetite.

Tabela 6. Fatores da Análise Fatorial de Correspondência

\begin{tabular}{|c|c|c|c|c|c|c|c|c|c|c|c|}
\hline \multicolumn{6}{|c|}{ Fatar 1} & \multicolumn{6}{|c|}{ Fatar 2} \\
\hline \multicolumn{3}{|c|}{ Fálo Fociesvo } & \multicolumn{3}{|c|}{ Pálo Negativo } & \multicolumn{3}{|c|}{ Pálo Positive } & \multicolumn{3}{|c|}{ Pálo Negative } \\
\hline varizues & c.c & $x^{2}$ & Vonínes & c $x$ & $x^{2}$ & Varisueis & cx & $\boldsymbol{x}^{2}$ & veríneis & cx & $x_{2}$ \\
\hline Homem & 4.2 & 48 & Itrukner & 35.4 & 48 & Iturer & 54 & 52 & Hamem & 6.4 & 52 \\
\hline Trabahis & 36 & 69 & Who Trabalns & 243 & $\infty 9$ & Trabaha & 236 & 31 & Who Trabaln & 16 & 31 \\
\hline Falawras & c.c & $x^{2}$ & Palantas & $c x$ & $x^{2}$ & Fallarsas & $c x$ & $\boldsymbol{x}^{2}$ & Pslantas & cx & $x^{2}$ \\
\hline insiedade & 112 & 094 & Lembarupas & 115 & 0.67 & Doerças & 158 & 0.83 & Droges & 15.4 & 0.89 \\
\hline Tristem & 9.4 & 0.82 & Arrepentimento & 8.4 & 099 & Iroute & 133 & 0.49 & Dificil-parar & 50 & 0.78 \\
\hline Desonfibo & 5.1 & 084 & Famitin & 6.6 & 090 & Lembrarças & 82 & 0.33 & Lberaide & 3.4 & 0.78 \\
\hline Barato & 5.1 & 084 & Comer & 19 & 059 & Thistem & 29 & 0.18 & Abotinêncin & 30 & 081 \\
\hline Fum & 2.7 & 10 & & & & Camer & 19 & & Perdas & 19 & 094 \\
\hline Recílin & 2.3 & 0.84 & & & & & & & Iffudar & 1.7 & 0.78 \\
\hline Desespero & 2.3 & 099 & & & & & & & Apjiertar & 1.7 & 0.78 \\
\hline \multirow[t]{2}{*}{ Sexo } & 1.0 & 0.84 & & & & & & & Anto-estima & 1.7 & 0.78 \\
\hline & & & & & & & & & $\operatorname{Pax}$ & 1.4 & 082 \\
\hline
\end{tabular}


O segundo fator apresenta a noção de que as drogas representam liberdade, porém podem trazer doenças, tristeza e até mesmo a morte, por isso é necessário ter força para mudar e encontrar a paz. Um primeiro pólo ligado a este fator, ao qual se associaram as variáveis Mulher e Trabalha, fala das lembranças da tristeza, das doenças, associando drogas com morte. Um segundo pólo, ao qual se associaram as variáveis Homem e Não trabalha aponta para a dificuldade de parar de usar, mas refere que para agüentar firme é preciso ter uma boa auto-estima.

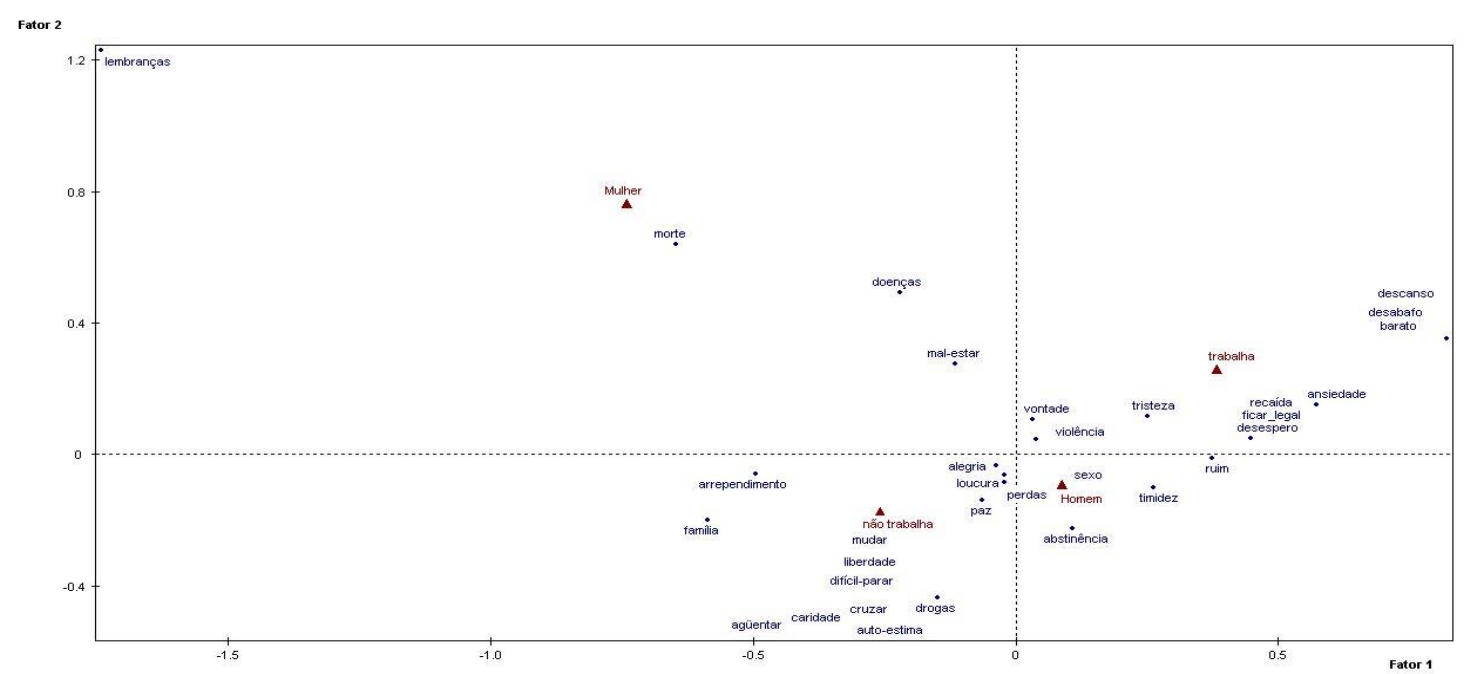

Figura 4. Projeção no eixo fatorial

A partir da projeção gráfica dos dois primeiros fatores no eixo fatorial (Figura 4), observa-se que o primeiro eixo traduz representações dos homens que trabalham, sobre álcool e outras drogas, associando-as a sexo, perdas, timidez, abstinência e violência. Estes campos se opõe no plano fatorial aos do segundo eixo, sobre o qual se projetam as representações das mulheres, que não trabalham, com relação ao álcool e outras drogas, aproximando-as dos campos morte e doenças.

\section{Discussão}

Este estudo procurou investigar as representações sociais do álcool e outras drogas de usuários de CAPS ad, bem como suas vulnerabilidades específicas com relação às DST/HIV/Aids, através de suas atitudes frente o uso do preservativo, uso do mesmo, motivos do não uso e conhecimento sobre HIV/Aids.

Com relação aos participantes da pesquisa, usuários do serviço do CAPS ad do município de Florianópolis, observou-se que a clientela predominante deste serviço é do sexo masculino, adulta, de baixa 
escolaridade e desempregada. Tais dados também foram encontrados em pesquisa de caracterização dos serviços de atenção à dependência de álcool e outras drogas em Florianópolis, no qual Spohr, Leitão e Schneider (2006) observaram predomínio masculino na clientela da maioria dos serviços do município.

De acordo com as autoras, em relação ao predomínio do sexo masculino, sabe-se que historicamente os problemas de consumo abusivo de álcool e drogas são mais comuns entre os homens. Há no município serviços que atendem ao público feminino, mas geralmente em instituições mistas (como é o caso do CAPS ad), porém quase não existem serviços especializados somente para a atenção ao público feminino, que tem se tornado cada vez mais vulnerável à dependência de álcool e outras drogas, o que modifica o cenário de décadas anteriores. Há estudos (SENAD, 2004) que mostram a necessidade de planejar instituições especializadas para esse público, pois ele tem suas especificidades, que devem ser levadas em consideração, para maior eficácia nas intervenções.

Outra característica dos atendimentos deste CAPSad tem sido atender em maior proporção munícipes de Florianópolis, porém também tem atendido em grande escala pessoas de cidades vizinhas, fato que pode ocorrer em função de ser o único CAPS ad da região até o momento da pesquisa ${ }^{2}$.

Com relação a baixa inserção no mercado de trabalho pelos usuários do CAPSad, estudo anterior realizado no município (SPOHR; LEITÃO; SCHNEIDER, 2006) também levantou que a categoria predominante nos diversos serviços voltados ao atendimento ao álcool e outras drogas foi a de desempregados, seguida pela de biscateiros. Este dado aponta para a necessidade de que estes serviços se debrucem sobre a importância da promoção de ações voltadas à reinserção social dos usuários. Uma das dificuldades para esta reinserção pode estar associada à baixa escolaridade dos usuários, visto que maior proporção dos mesmos $(48,3 \%)$ declarou ter o ensino fundamental incompleto, e 3,4\% declarou não ter estudado.

A reinserção social torna-se, portanto, aspecto primordial para que se possa consolidar a melhoria da qualidade de vida dos usuários de uma forma integral. Indivíduos com problemas de saúde mental geralmente necessitam, para reinserirem-se na comunidade, de apoio social. Assim, o objetivo final do cuidado em saúde, em sua vertente social, é mais do que o tratamento dos sintomas e sim a reintegração das pessoas à vida em sociedade, pois os indivíduos integrados às suas comunidades tendem a viver mais e podem ter maiores recursos para lidar e/ou recuperar-se do sofrimento físico, psíquico, social que afetam sua saúde. É necessário, portanto, que o cuidado em saúde considere os usuários como seres sociais que compartilham de um contexto complexo (MCDOWELL; NEWELL, 1996). 
Quanto às substâncias utilizadas pelos usuários, observou-se a predominância pelo uso de várias $(56,3 \%)$, em segundo lugar ficou a declaração do uso do álcool $(25,3 \%)$, em terceiro a maconha, com $13,8 \%$ e em quarto lugar ficou o crack, com 4,6\%. Estes dados corroboram dados de outras pesquisas, que demonstram o grande percentual do uso de álcool pela população em geral (CARLINI et al., 2007).

No que concerne aos relacionamentos amorosos, a maior proporção $(57,5 \%)$ declarou não ter parceiro fixo no momento da pesquisa, enquanto $42,5 \%$ disse ter um parceiro fixo. Porém paradoxalmente, com relação ao número de parceiros sexuais, observou-se que a maior proporção $(47,7 \%)$ afirmou ter tido relações sexuais com a mesma pessoa nos últimos 12 meses, enquanto $40,7 \%$ afirmou ter tido relações com mais de uma pessoa.

E apesar do grupo ter se posicionado favoravelmente frente ao uso do preservativo, a adesão ao uso consistente do mesmo foi baixa, pois apenas $36,1 \%$ declarou ter utilizado em todas as relações sexuais dos últimos 12 meses, enquanto 34,9\% declararam não ter utilizado, $20,5 \%$ declararam ter utilizado em algumas relações e $8,4 \%$ declarou não saber se utilizou.

O principal motivo para a não utilização do preservativo, com $63 \%$ das respostas, foi a confiança no parceiro. Tal dado torna-se um paradoxo frente ao percentual dos que declararam ter parceiro fixo. Outro dado importante é que $10 \%$ dos participantes afirma que não utilizou preservativos porque estava sob efeito de álcool ou outras drogas.

Outro dado que parece fazer sentido frente à baixa adesão do uso do preservativo é que considerável proporção $(24,1 \%)$ dos participantes afirma já ter tido uma DST enquanto $21,8 \%$ diz não saber se teve. Esta porcentagem é superior a encontrada em estudo sobre prevalência geral de DST entre a população adulta no Brasil, que observou a prevalência de sintomas de DST em 13,5\% dos participantes (CARRET; FASSA; SILVEIRA; BERTOLDI; HALLAL, 2004).

Perguntou-se se os participantes se eles se consideram bem informados sobre aids. A maior proporção $47,1 \%$ afirmou que se considera bem informado, enquanto $26,4 \%$ não se considera bem informado e $26,4 \%$ não sabe. Estes resultados são coerentes com o score obtido pelos participantes no sub-teste de conhecimento sobre a transmissão do vírus HIV (dimensão vírus e transmissão da aids) de Camargo, Barbará e Bertoldo (2005). A média de conhecimento do grupo foi de 5,12 ( $D P=2,93$ ) dentre 10 possíveis; Tal média de acerto se encontra abaixo da nota de corte de 7 acertos do sub-teste vírus da aids e transmissão da doença, parte integrante da medida sobre conhecimento acerca de aids, o que indica um baixo índice de 
conhecimento frente a doença. Além disso, porcentagem importante $(26,4 \%)$ afirmou não saber o que é uma DST.

Com relação às representações sociais dos usuários do CAPSad sobre álcool e outras drogas observa-se a representação social destas substâncias ligada à alegria, festas, diversão, e apesar da associação com as perdas e danos também surge a importante ligação com o sexo. Considerando que o uso destas substâncias também foi citado como motivo para a não utilização do preservativo, ressalta-se a importância de pensar ações de prevenção das DST/HIV/Aids entre esta população.

Essa representação social da droga associando-a ao sexo parece fazer sentido entre estes participantes, pois apesar de terem tido atitudes favoráveis frente o uso do preservativo, na prática este uso não foi consistente, além da alta prevalência de DST entre os mesmos. A relação entre as representações sociais e as atitudes é possível, uma vez que as atitudes impregnam as representações sociais e estas últimas determinam as condutas desejáveis ou permitidas. A compreensão das representações sociais proporciona o acesso às justificativas utilizadas para orientar julgamentos e ações acerca do mundo e permite conhecer as estratégias dos seres humanos quando estão diante da tarefa de compreender fatos desconhecidos. Assim, reconstroem-se as situações como conhecimentos que regem 0 processo de comunicação e ação em relação aos fenômenos (MOSCOVICl, 1978).

Uma vez considerado que as representações sociais e as práticas estão estreitamente ligadas, determinando não só comportamentos distintos, como também atitudes específicas diante de um problema (ABRIC, 2001), o sentido que os indivíduos atribuem à aids pode influenciar na retenção de informações preventivas e, conseqüentemente, na adesão às práticas de prevenção.

Além disso, apesar da amostra ter maior proporção de homens, observou-se uma importante diferenciação nas representações do álcool e outras drogas de homens e de mulheres. Enquanto para eles existe uma associação com prazer, sexo e desespero, para elas, surge à noção do arrependimento frente às lembranças, e da preocupação com as conseqüências causadas na família. Isto ocorre porque as posições dos indivíduos com relação à cultura, ao gênero, idade e classe, entre outros, constituem elementos determinantes para a compreensão de comportamentos frente aos diversos objetos, como por exemplo, álcool e outras drogas e ao risco de infecção pelo HIV.

Ser homem, portanto, significaria soberania, não revelar sentimentos e arriscar sempre, principalmente com relação à sexo (GARCIA, 1998). E ser mulher, associa-se à passividade, submissão, cuidado e temor em relação ao homem, valorização da família, resignação ao papel cristalizado de objeto do controle masculino (MOTA, 1998). 
Esta desigualdade de poder nas relações entre homens e mulheres é um dos motivos da dificuldade que ambos têm em discutir formas seguras de exercer a sexualidade (VILLELA, 1998; GIACOMOZZI; CAMARGO, 2004), expondo-os por conseqüência às DST e à aids.

\section{Considerações Finais}

Observou-se que esta população apresentou dados de vulnerabilidade às DST/HIV/Aids, pois apesar de uma atitude favorável ao uso do preservativo, na prática houve uma baixa adesão ao uso do mesmo, baixo conhecimento sobre transmissão do HIV e importante presença de DSTs em suas histórias de vida, bem como uma grande quantidade de participantes que afirma não ter conhecimento do que é uma DST.

Além disso, observaram-se especificidades com relação a vulnerabilidade dos usuários do CAPSad com relação ao HIV/Aids, pois observou-se que o estar sobre efeito de alguma substância psicoativa foi fator dificultador da utilização do preservativo. Por outro lado, nas evocações sobre álcool/drogas surge a importante associação destas com sexo, diversão e prazer, o que indica que a combinação drogas/álcool pode ser igual a sexo desprotegido.

Tal relação faz sentido a partir de outros estudos que também observaram esta ligação entre sexo, drogas e prazer (SISSA, 1999; BERGERET; LEBLANC, 1991) Tais autores observaram que as drogas, assim como o sexo, se relacionam com fonte de satisfação, de sensação agradável que inclui, uma dimensão de prazer, sem a qual seria inexplicável a atração por ela exercida sobre jovens e adultos. É necessário, portanto que a dimensão do prazer seja considerada, tanto em trabalhos preventivos, quanto em trabalhos onde a redução de danos é a temática principal, para que se possa dialogar a partir da verdade de cada sujeito a respeito da temática.

Além disso, mais especificamente sobre a questão das DST/HIV/Aids, faz-se fundamental propor oficinas que discutam sexualidade, prazer e sexo seguro, para usuários de CAPSad, pois a prevenção e o diagnóstico destes agravos deve ser realizada o mais precocemente possível.

\section{Referências}

ABRIC, J. C. O estudo experimental das representações sociais. In: JODELET, D. (Org.). As representações sociais. Rio de Janeiro: EdUERJ, 2001, p. 155-172.

AYRES, J. R. C. M.; FRANÇA, JR, I.; CALAZANS, G. J.; SALETTI FILHO, H. C. Vulnerabilidade e prevenção em tempos de Aids. In: 
BARBOSA, M. R.; PARKER, R. (Orgs.). Sexualidade pelo avesso: direitos, identidades e poder. Rio de Janeiro: IMS/UERJ, 1999, p. 4972.

AZEVEDO, R. C. S.; BOtEGA, N. J.; GUI MARAES, L. A. M. Crack users, sexual behavior and risk of HIV infection. Revista Brasileira de Psiquiatria, São Paulo, v. 29, n. 1, p. 26-30, mar. 2007.

BARBARÁ, A. S. B.; SACHETTI, V.; CREPALDI, M. A. Contribuições das representações sociais ao estudo da AIDS. Interação em Psicologia, Curitiba, v. 9, n. 2, p. 331-339, 2005.

BERGERET, J; LEBLANC, J. Toxicomanias: uma visão multidisciplinar. Porto Alegre: Artes Médicas, 1991.

CAMARGO, B. V.; BARBARÁ, A.; BERTOLDO, R. B. Um instrumento de medida da dimensão informática da representação social da aids. In:

IV Jornada Internacional e II Conferência Brasileira sobre Representações Sociais, 4., 2005, João Pessoa, 2005, p. 13851395.

CAMARGO, B. V; BOTELHO, L. J. Aids, sexualidade e atitudes de adolescentes sobre proteção contra o HIV. Revista de Saúde Pública, São Paulo, v. 41, n. 1, fev. 2007.

CAMARGO, B. V.; BARBARA, A. S. B. Efeito de panfletos informativos sobre a Aids em adolescentes. Psicologia Teoria e Pesquisa, Brasília, v. 20, n. 3, p. 279-287, set./dez. 2004.

CAMARGO, B. V.; BERTOLDO, R. B. Comparação da vulnerabilidade em relação ao HIV de estudantes da escola pública e particular. Estudos de Psicologia, Campinas, v. 23, n. 4, p. 369-379, out./dez. 2006.

CARLINI, E. A; GALDUROZ, J. C.; NOTO, A. R. II Levantamento Domiciliar sobre uso de drogas psicotrópicas no Brasil: estudo envolvendo as 108 maiores cidades do país - 2005. Brasília: Secretaria Nacional Antidrogas, 2007.

CARRET, M. L. V.; FASSA, A. G.; SILVEIRA, D. S.; BERTOLDI, A. D.; HALLAL, P. C. Sintomas de doenças sexualmente transmissíveis em adultos: prevalência e fatores de risco. Revista de Saúde Pública, São Paulo, v. 38, n. 1, p. 76-84, fev. 2004.

COOK, R. L.; POLLOCK, N. K.; RAO, A. D.; CLARK, D. B. Increased prevalence of herpes simplex virus type 2 among adolescent women with alcohol disorders. Adolescent Health, San Francisco, v. 30, n. 1, p. 169-74, mar. 2002.

DOISE, W.; CLEMENCE, A.; LORENZI-CIOLDI, F. Représentations sociales et analyses de donnés. In: COUTINHO, M. P. L., LIMA, A. S. (Orgs.). Representações Sociais: abordagem interdisciplinar. J oão Pessoa: Ed. Universitária/UFPB, 1992.

FONSECA, A. A.; AZEVEDO, R. L. W.; ARAUJ O, L. F. Representações sociais de universitários de psicologia acerca da maconha. Estudos de Psicologia, Campinas, v. 24, n. 4, p. 441-449, out./dez. 2007. 
Garcia, S. M. Conhecer os homens a partir do gênero e para além do gênero. In: ARILHA, M.; RIDENTI, G. U.; MEDRADO, B.; (Orgs.). Homens e masculinidades. Outras palavras. São Paulo: Editora 34, 1998, p. 31-50.

GIACOMOZZI, A. I. Variáveis Culturais e Psicossociais associadas à vulnerabilidade étnica ao HIV/Aids: estudo comparativos entre Brasil e França. 2008. Tese (Doutorado em Psicologia) - Programa de Pós-graduação em Psicologia, Universidade Federal de Santa Catarina, Florianópolis, 2008.

GIACOMOZZI, A. I.; CAMARGO, B. V. Eu confio no meu marido: estudo da representação social de mulheres com parceiro fixo sobre a prevenção da AIDS. Psicologia: Teoria e Prática, São Paulo, v. 6, n. 4, p. 31-44, 2004.

LEBART, S.; SALEM, A. L'analyse statistique de données textuelles. Paris: Bordas, 1988.

LIMA, M. L. P. Atitudes. In: VALA, J.; MONTEIRO, M. B. (Orgs.). Psicologia social. Lisboa: Fundação Calouste Gulbenkian, 1996, p.197-199.

MINISTÉRIO DA SAÚDE. Secretaria de Políticas de Saúde. Projeto ajude-Brasil: avaliação dos usuários de drogas injetáveis dos projetos de redução de danos apoiados pela CN-DST/ AIDS. Brasília: Secretaria de Políticas de Saúde, 2001.

MINISTÉRIO DA SAÚDE. A política do Ministério da Saúde para atenção integral à usuários de álcool e outras drogas. Série $B$ textos básicos de saúde. Brasília: Ministério da Saúde, 2003.

$\mathrm{MOSCOVICl}, \mathrm{S}$. La psychanalise son image et son publique. Paris: Presses Universitaires de France, 1981.

MOTA, M. P. Gênero e sexualidade: fragmentos de identidade masculina nos tempos de AIDS. Cadernos de Saúde Pública, v. 1, n. 14, p. 145-55, 1998.

MURRAY, C. J. L.; LOPEZ, A. D. The global burden of disease: a comprehensive assessment of mortality and disability, form diseases, injuries and risk factors in 1990 and projected to 2020. Global Burden of Disease and I njury Series, Cambridge, Massachusetts Harvard School of Public Health to World Health Organization and World Bank, v. I, 1996.

OLIVEIRA, A.; AMÂNCIO, L. A. Análise factorial de correspondências no estudo das representações sociais - as representações sociais da morte e do suicídio na adolescência. In: MOREIRA, A. S. P.; CAMARGO, B. V.; JESUÍNO, J. C.; NÓBREGA, S. M. (Orgs.). Perspectivas teórico-metodológicas em representações sociais. J oão Pessoa: UFPB Editora Universitária, 2005, p. 323-362. ORGANIZAÇÃO MUNDIAL DA SAÚDE. Relatório sobre a saúde no mundo 2001 - saúde Mental: nova concepção, nova esperança. Genebra: OMS, 2001. 
MARKOVÁ, I. Dialogicidade e representações sociais: as dinâmicas da mente. Petrópolis: Vozes, 2006.

MARTINS, L. B.; COSTA-PAIVA, L.; OSIS, M.; SOUZA, M.; PINTONETO, A.; TADINI, V. Fatores associados ao uso de preservativo masculino e ao conhecimento sobre DST/AIDS em adolescentes de escolas públicas e privadas do Município de São Paulo, Brasil. Cadernos de Saúde Pública, Rio de Janeiro, v. 22, n. 2, p. 315323, 2006.

MADEIRA, M. Representações sociais e educação: importância teórico-metodológica de uma relação. In: MOREIRA, A. S. P. (Org.). Representações sociais: teoria e prática. João Pessoa: Universitária, 2001, p.123-132.

MOSCOVICl, S. A representação social da Psicanálise. Rio de Janeiro: Zahar Editores, 1978.

MCDOWELL, I.; NEWELL, C. Social Health. In: MCDOWELL, I; NEWELL, C. (Orgs.). Measuring health: a guide to rating scales and questionnaires. 2 ed. New York: Oxford University, 1996, p. 122-76.

RODRIGUES, A.; ASSMAR, E. M. L.; JABLONSKI, B. Psicologia social. Petrópolis: Vozes, 2002.

SCIVOLETTO, S; TSUJI, R. K.; ABDO, C. H. N.; QUEIROZ, S.; ANDRADE, A. G.; GATTAZ, W. F. Relação entre consumo de drogas e comportamento sexual de estudantes de segundo grau de São Paulo. Revista Brasileira de Psiquiatria, São Paulo, v. 21, n. 2, p. 87-94, abr/jun.1999.

SISSA, G. O prazer e o mal: filosofia da droga. Rio de Janeiro: Civilização Brasileira, 1999.

TAQUETTE, S.; ANDRADE, R. B.; VILHENA, M.; PAULA, M. A relação entre as características sociais e comportamentais da adolescente e as doenças sexualmente transmissíveis. Revista da Associação Médica Brasileira, São Paulo, v. 51, n. 3, p. 148-152, jun. 2005.

TAPERT, S. F.; AARONS, G.; SEDLAR G.; BROWN, S. A. Adolescent substance use and sexual risk-taking behavior. Journal of Adolescent Health, San Francisco, v. 29, n. 1, p. 181-189, maio 2001.

SPOHR, B.; LEITÃO, C.; SHNEIDER, D. Caracterização dos serviços de atenção à dependência de álcool e outras drogas na região da Grande Florianópolis. Revista de Ciências Humanas, Florianópolis, EDUFSC, n. 39, p. 219-236, abr. 2006.

SECRETARIA NACIONAL ANTIDROGAS (SENAD). Atualização de conhecimentos sobre redução da demanda de drogas. Brasília: SENAD, 2004.

VALA, J. Representações sociais e Psicologia Social do conhecimento cotidiano. In: VALA, J.; MONTEIRO, B. (Orgs.). Psicologia Social. Lisboa: Fundação Calouste Gulbenkian, 2000, p. 457-502.

VILLELA, W. Homem que é homem também pega AIDS? In: ARILHA, M.; RIDENTI, G. U.; MEDRADO, B. (Orgs.). Homens e 
masculinidades. Outras palavras. São Paulo: Editora 34, 1998, p. 129-42.

\section{Endereço para correspondência}

Andréia I sabel Giacomozzi

Rua Felipe Shimidt, 1210, Edifício Maison Classic, apto 1206, CEP 88010-002, Florianópolis - SC, Brasil.

Endereço eletrônico: agiacomozzi@hotmail.com

Recebido em: 15/04/2010

Reformulado em: 12/08/2010

Aceito para publicação em: 16/08/2010

Acompanhamento do processo editorial: Adriana Benevides Soares

\section{Notas}

* Psicóloga, Mestre e Doutora em Psicologia pela UFSC.

${ }^{1}$ Disponível em <www.unodc.org.br>.

${ }^{2}$ Após a realização desta pesquisa, foi inaugurado mais um CAPSad no município de Florianópolis. 